\title{
Acute Appendicitis in a Diabetic Child with Salmonella Infection
}

\section{Stylianos Roupakias, Maria-loanna Apostolou, Anastasia Anastasiou}

Pediatric Clinic, General Hospital Volos, Volos, Greece

Received January 8, 2020; Accepted January 28, 2021.

Key words: Appendicitis - Salmonella - Diabetes - Children

Abstract: Acute appendicitis is the most frequent and challenging condition requiring emergent intrabdominal surgery in children. The diagnosis of appendicitis becomes more difficult and challenging in children, especially with other medical problems. Computed tomography is the primary tool for diagnosing or excluding appendicitis in cases with atypical presentation. Salmonella infections may present as acute abdominal problems in children. We present a clinical combination that has never been previously reported, of a diabetic girl with non-typhoid Salmonella infection, diagnosed with acute appendicitis. We wonder about the causal correlation of these diseases, versus their simple coexistence.

Mailing Address: Stylianos Roupakias, MD., H'Argyri 74 str., 38221, Volos, Greece; Phone: +302 421031 020; e-mail: stylroup@yahoo.gr 


\section{Introduction}

Acute appendicitis is one of the most common causes of abdominal pain and is the most frequent condition requiring emergent intrabdominal surgery in children. Yet, despite its common occurrence, it still remains a continuing clinical and diagnostic challenge, especially in the pediatric population, due to potential atypical clinical presentation in this age group, and also a wide range of differential diagnoses (Singh et al., 2014), including gastroenteritis. The early diagnosis is vital for the successful outcome because the delay in diagnosis ( $>36-48$ hours) can lead to gangrene or perforation and associated complications with increased morbidity prolonged hospitalization and even mortality in young children (Singh et al., 2014), especially those with coexisting diseases. Advanced radiologic imaging has an everincreasing role in the prompt and accurate diagnosis of acute appendicitis in the pediatric population with the use of computed tomography (CT) proposed from some authors as the primary tool for diagnosing or excluding appendicitis in children (Callahan et al., 2002), improving also the rupture rates.

We present a diabetic girl with non-typhoid Salmonella infection and acute appendicitis, a clinical combination that has never been previously reported.

\section{Case report}

A 6-year-old girl presented to the emergency department with a 24-hour history of central abdominal pain, eight episodes of loose stool, two episodes of vomiting and fever up to $38.2{ }^{\circ} \mathrm{C}$. Two-days prior, her pediatrician prescribed amoxicillin plus clavulanic acid for suspected acute otitis media. The girl had a medical history of diabetes mellitus type I treated with short-acting and long-acting insulin, asthma and afebrile seizures treated with valproic acid. On physical examination, the patient's tonsils were mildly swollen, red with whitish spots and an otoscopic examination revealed turbidity of the left tympanic membrane. Her abdomen was tender in all four quadrants especially in the right lower one (RLQ) and bowel sounds were present. Blood tests were performed and they revealed a white cell count (WCC) of 5,200 K/ $\mu$ l (types of WBCs: neut.: 70.30\%, lymp.: 12.70\%, mono: 16.80\%) and C-reactive protein (CRP) of $193.52 \mathrm{mg} / \mathrm{I}$ (normal range: $0-5 \mathrm{mg} / \mathrm{l}$ ). Stool samples were requested and a blood culture was taken along with a blood gas analysis. Intravenous fluids and antibiotics (ampicillin) were administered to her. Approximately 12 hours later, her abdominal pain was getting worse and she had another four episodes of loose stool. Blood tests were repeated and they showed an increase of C-reactive protein (CRP) up to $243.09 \mathrm{mg} / \mathrm{I}$ with a white cell count $(\mathrm{WCC}$ ) of $6,800 \mathrm{~K} / \mu \mathrm{l}$ (types of WBCs: neut.: $50 \%$, lymp.: $10 \%$, mono: $5 \%$ ). An abdominal ultrasound was performed that showed the presence of free intraperitoneal fluid surrounding the loops of the bowel, slightly echovoid in scattered areas, and swelling of the colon along with increased echogenicity of the surrounding fat. Due to these findings, her antibiotic therapy was altered to metronidazole and cefotaxime and surgical assessment was requested. After a 
recommendation for further imaging, a computed tomography of the abdomen and pelvis was performed that revealed acute appendicitis, so an open appendectomy was scheduled. At surgery, an inflammation-oedema of all the body and the tip of the appendix was found and an excessive amount of free straw coloured reactive intraperitoneal fluid. It was an inflamed without necrosis appendicitis, according to clinical-imaging-operative-clinical AAST grade system. The girl became afebrile and painless immediately after the surgery. In the meantime, the results from her stool cultures showed that she had non-typhoid Salmonella resistant to the usually recommended regimens (ampicillin, amoxicillin plus clavulanic acid, cefaclor or trimethoprim-sulfamethoxazole). She was discharged three days later, showing remarkable clinical improvement, with instructions for per os antibiotic treatment with ampicillin plus sulbactame according to the chemotherapy sensitivity test. Ten days after surgery, she had a new positive for Salmonella stool culture. So, per os ciprofloxacine was recommended and stopped with a new negative stool culture 7 days later.

\section{Discussion}

Salmonella infections may present as acute abdominal problems in children, can be particularly challenging and lead to surgical emergency (Arda et al., 2001; Kumar et al., 2015). The spectrum of abdominal manifestations from Salmonella (typhi and paratyphi) infections includes intestinal perforations, acute cholocystitis, salpigitis, and rarely appendicitis (Kumar et al., 2015; Stewart-Parker et al., 2016). Nontyphoid Salmonella species cause gastroenteritis and are also rarely associated with appendicitis (Stewart-Parker et al., 2016). In most cases Salmonella pathogens cause an appendicitis-mimicking syndrome through infectious ileocolitis and/or mesenteric lymphadenitis called as pseudoappendicitis which can lead to a needless operation (Kumar et al., 2015; Stewart-Parker et al., 2016). There are however a few reported cases of acutely inflamed or perforated appendicitis as a result of Salmonella infection (Stewart-Parker et al., 2016). Salmonella appendicitis may require different antibiotic treatment to that used in appendicitis, so preoperarive stool cultures and bacterial analysis of peritoneal fluid may facilitate prompt and appropriate management (Stewart-Parker et al., 2016). Even if there is not a precise diagnosis in Salmonella related acute abdomen even with abdominal ultrasound sonography and/or computerized tomography, the surgical approach is the right choice, considering the high morbidity associated with delayed treatment of appendicitis (Arda et al., 2001; Manganaro et al., 2006), despite a relative risk of a needless operation. A positive preoperative culture for Salmonella should not contraindicate appendectomy (Stewart-Parker et al., 2016).

Furthermore, the diagnosis of appendicitis becomes more difficult and challenging in children with other medical problems, including diabetes mellitus and the associated relative immunodeficiency (Stewart et al., 2014). Common and rare 
infectious diseases, including those of the gastrointestinal track, are more frequent and serious in the diabetic population (Tsai et al., 2008; Casqueiro et al., 2012). Patient who are medication-dependent diabetics are more likely to develop infection from some Salmonella species (Telzak et al., 1991). Diabetics present a more complicated, atypical or clouding clinical picture of acute appendicitis having a higher risk for delayed diagnosis, perforation and postoperative complications (Stewart et al., 2014; Bach et al., 2016). Abdominal pain in diabetic patients necessitating a higher index of suspicion (Bach et al., 2016), early surgical consultation (Tsai et al., 2008), and more aggressive decision for operation.

An appendiceal culture is not a routine performed test in surgically treated patients with acute appendicitis, especially without evidence of a purulent exudate in the lumen. Non-typhoid Salmonella caused appendicitis may indeed be under-reported as peritoneal fluid is not universally sent for examination at the time of surgery, and laboratories often do not fully identify coliforms isolated from peritoneal fluids, assuming that these are gut flora (Stewart-Parker et al., 2016).

\section{Conclusion}

In summary, Salmonella infections are a rare cause of appendicitis. Children with atypical presentations should be observed closely. Stool samples should be sent to all patients with loose stool and suspected appendicitis, and early surgical consultation is recommended especially in immunocompromised patients. Should a positive stool culture for Salmonella in a patient with identical CT/clinical and operative findings of appendicitis, be considered a strong evidence of Salmonella infection involvement as the real cause of appendicitis against their simple co-existence?

\section{References}

Arda, I. S., Ergin, F., Varan, B., Demirhan, B., Aslan, H., Ozyaylali, I. (2001) Acute abdomen caused by Salmonella typhi murium infection in children. J. Pediatr. Surg. 36(12), 1849-1852.

Bach, L., Donovan, A., Loggins, W., Thompson, S., Richmond, B. (2016) Appendicitis in diabetics: Predictors of complications and their incidence. Am. Surg. 82(8), 753-758.

Callahan, M. J., Rodriguez, D. P., Taylor, G. A. (2002) CT of appendicitis in children. Radiology 224(2), 325-332.

Casqueiro, J., Casqueiro, J., Alves, C. (2012) Infections in patients with diabetes mellitus: A review of pathogenesis. Indian J. Endocrinol. Metab. 16(1), 27-36.

Kumar, K. J., Chavan, A., Deb, P., Manoli, P. (2015) Acute abdomen caused by typhoid fever mimicking acute appendicitis. MJPCH $\mathbf{2 1 ( 4 ) , 1 - 3 . ~}$

Manganaro, A., Impellizzeri, P., Manganaro, A., Cutrupi, A., Formica, I., Zuccarello, B. (2006) Acute abdomen caused by Salmonella typhi acute appendicitis. Minerva Pediatr. 58(2), 203-205.

Singh, M., Kadian, Y. S., Rattan, K. N., Jangra, B. (2014) Complicated appendicitis: Analysis of risk factors in children. Afr. J. Paediatr. Surg. 11(2), 109-113.

Stewart, C. L., Wood, C. L., Bealer, J. F. (2014) Characterization of acute appendicitis in diabetic children. J. Pediatr. Surg. 49(12), 1719-1722. 
Stewart-Parker, E. P., Atta, M., Doddi, S. (2016) A curious cause of appendicitis. BMJ Case Rep. 2016, bcr2016216150.

Telzak, E. E., Greenberg, M. S., Budnick, L. D., Singh, T., Blum, S. (1991) Diabetes mellitus - A newly described risk factor for infection from Salmonella enteritidis. J. Infect. Dis. 164(3), 538-541.

Tsai, S. H., Hsu, C. W., Chen, S. C., Lin, Y. Y., Chu, S. J. (2008) Complicated acute appendicitis in diabetic patients. Am. J. Surg. 196(1), 34-39. 